\title{
The effectiveness of two different multimodal training modes on physical performance in elderly
}

L'udmila Oreská ${ }^{1, \#, ~ L u c i a ~ S l o b o d o v a ́ 4,5 \#, ~ M a t e j ~ V a j d a ~}{ }^{1}$, Adriana Kaplánová ${ }^{2}$, Veronika Tirpáková6, Ján Cvečka ${ }^{3}$, Gabriel Buzgó ${ }^{1}$, Jozef Ukropec ${ }^{4}$, Barbara Ukropcová ${ }^{1,4,5 \&}$, Milan Sedliak $^{1, \&}$

(1) Department of Biological and Medical Sciences, Faculty of Physical Education and Sports, Comenius University, Bratislava, Slovakia; (2) Department of Sport Sciences in Educology and Humanities, Faculty of Physical Education and Sports, Comenius University, Bratislava, Slovakia; (3) Diagnostic Centre of Professor Hamar, Faculty of Physical Education and Sports, Comenius University, Bratislava, Slovakia; (4) Institute of Pathophysiology, Faculty of Medicine, Comenius University, Bratislava, Slovakia; (5) Institute of Experimental Endocrinology, Biomedical Research Centre, Slovak Academy of Sciences, Bratislava, Slovakia; (6) Institute of Sports Medicine, Faculty of Medicine, Slovak Medical University, Bratislava, Slovakia. (\#) shared first authorship; (\&) shared last authorship

This article is distributed under the terms of the Creative Commons Attribution Noncommercial License (CC BY-NC 4.0) which permits any noncommercial use, distribution, and reproduction in any medium, provided the original author(s) and source are credited.

\begin{abstract}
The study compared the effect of 12-week multimodal training programme performed twice a week at the regular exercise facility (REF) with the 12-week multimodal training programme performed three times per week as a part of the research programme (EX). Additionally, the study analysed how the experimental training programme affect the physical performance of cognitive healthy and mild cognitive impaired elderly (MCI). The REF training group included 19 elderly $(65.00 \pm 3.62$ years). The experimental training programme combined cognitively healthy (EXH: $n=16 ; 66.3 \pm 6.42$ years) and age-matched individuals with MCI (EXMCI: $n=14$; $66.00 \pm 4.79$ years). $10 \mathrm{~m}$ maximal walking speed (10mMWS), Five Times Sit-to-Stand Test (FTSS), maximal and relative voluntary contraction (MVC \& rel. MVC) were analysed. The REF group improved in 10mMWS $(\mathrm{t}=2.431, \mathrm{p}=.026)$, the $\mathrm{MVC}(\mathrm{t}=-3.528, \mathrm{p}=.002)$ and relative $\operatorname{MVC}(\mathrm{t}=3.553, \mathrm{p}=.002)$. The EXH group improved in FTSS $(\mathrm{t}=5.210, \mathrm{P}=.000), \mathrm{MVC}(\mathrm{t}=2.771$, $\mathrm{p}=.018)$ and relative MVC $(\mathrm{t}=-3.793, \mathrm{p}=.004)$. EXMCI improved in FTSS $(\mathrm{t}=2.936, \mathrm{p}=.012)$ and $\operatorname{MVC}(\mathrm{t}=-2.276, \mathrm{p}=.040)$. According to results, both training programmes sufficiently improved walking speed and muscle strength in cognitively healthy elderly. Moreover, the experimental training programme improved muscle strength in MCI elderly.
\end{abstract}

Key Words: older adults, real-life implementation, multimodal exercise training, intention-totreat strategy.

Eur J Transl Myol 30 (1): xx1-x10, 202

\begin{abstract}
Ageing is associated with a progressive decline in physical performance, which is a powerful and independent risk factor for premature mortality. ${ }^{1}$ Physical performance is characterized as an ability to independently perform daily living activities without any difficulties. $^{2}$ Logically lower physical performance is accompanied with a higher rate of sarcopenia, body fat, impairment of cardiovascular and metabolic systems, and neurocognitive functions, which results in a general decrease of overall quality of life in elderly. ${ }^{3-6}$ This progressive deterioration of physical performance and neurocognitive functions is even more significant in the
\end{abstract}

elderly with cognitive impairment and early stages of dementia. Therefore, the increase of muscle mass, strength, flexibility, endurance and cardiorespiratory fitness, as essential components of physical performance, is very important, since when reduced, they can significantly contribute to the limited mobility, physical performance and cognitive state. ${ }^{1,2,9}$ There is good evidence that regular exercise improves physical performance in cognitively healthy elderly and individuals with mild cognitive impairment (MCI). Based on large research evidence regular exercise improves not only physical performance but also slows 
down or stabilizes the cognitive decline and improves the activation of structures in the brain, which are responsible for tasks requiring executive functions, attention, processing speed and memory in patients with MCI. ${ }^{2,7,10,11}$ However, the exercise type, dosing and length of the intervention are crucial of how long these effects would last. ${ }^{11}$ The American College of Sports Medicine and American Heart Association guidelines recommend resistance training with the intensity at 60 $70 \%$ of 1 repetition maximum (1RM) for $8-12$ repetitions on 2 or 3 non-consecutive days per week. ${ }^{11,12,13}$ The effective threshold for endurance exercise represents 150-300 minutes per week at moderate intensity or 75150 minutes per week at a vigorous intensity, at least three days a week. ${ }^{12,14}$ Besides, coordination training is prescribed 2-3 times per week in duration for 45-60 minutes, with the most challenging tasks performed 2-3 times per session. However, the evidence is largely based on expert opinion rather than research evidence. ${ }^{14,15}$ However, many elderly might find it challenging to comply with this exercise frequency. Thus, the combination of exercises mentioned above in two or three sessions per week might be a feasible and effective alternative for creating a suitable multimodal exercise programme. Nevertheless, it is unknown if the minimum frequency of multimodal training sessions twice per week is still sufficient to enhance the physical performance in the elderly who have had no previous experience with the regular exercise and are cognitively healthy or diagnosed with MCI., ${ }^{2,16}$ Within the study, we mainly aimed to compare the effectiveness of the 12 week multimodal, performed at the regular exercise facility (REF) twice per week, with the 12-week experimental multimodal training programme which is a part of our research programmes (EX) and is performed three times per week. The secondary aim was to compare the effectiveness of the experimental multimodal training between cognitively healthy elderly and age-matched elderly with MCI and how this training affects the indicators of physical performance such as walking speed and muscle strength.

\section{Materials and Methods}

\section{Ethical statement}

The study was approved by the Ethics Committees of University Hospital Bratislava, the Faculty of Physical Education and Sports Comenius University in Bratislava, and the Ethics Committee of the Bratislava Region Office.

The study protocol conforms to the ethical guidelines of the Declaration of Helsinki 2000, and its later amendments from 2013. All participants provided witnessed written informed consent prior to entering the study and their capacity to undergo exercise intervention was assessed by a cardiologist. The study was registered under the Clinical trials. gov registration number: NCT03330470.

\section{Participants}

In total, forty-nine active, cognitively healthy elderly and their counterparts with MCI were recruited (sex: $12 \mathrm{M} / 37 \mathrm{~F}$; age: $65.71 \pm 4.39$ years; weight: $79.82 \pm 17.28$ $\mathrm{kg}$ ). No previous history of exercise within the past five years was recorded. The exclusion criteria were the presence of severe mobility impairments, cardiovascular, neurological and other uncontrolled chronic diseases that might interfere with the training programme

\section{Study design}

Training programs were fully supervised by professional coaches with previous experience of exercising with elderly and supervising research training programmes. Cognitively healthy elderly were stratified into REF and EX groups and compared to age and body weightmatched individuals with MCI. They took part in two different 12-week multimodal resistance, endurance and aerobic-coordination training programmes, one of them emphasizing the Intention to Treat Strategy (REF) and the other performed within the Research Facility (EX). Physical performance tests included measurements of performance in $10 \mathrm{~m}$ maximal walking speed test (MWS), five times sit-to-stand test (FTSS) and measurements of maximal and relative voluntary contraction (MVC \& rel. MVC). The elderly were tested 14 days before and within two weeks after the last session of the exercise intervention. Both testing sessions were in similar timepoints, and the order of tests was strictly set. The tests were performed under the supervision of two or three researchers with previous testing experience. The baseline characteristics of participants are shown in Table 1. Additionally, we included the group of MCI elderly according to 1) Mini-Mental State Examination (MMSE, score 20-24) and 2) Montreal cognitive assessment, MoCA (score 22-25), a standard diagnostic test for MCI. ${ }^{17}$

Table 1. Baseline characteristics of participants

\begin{tabular}{ccccc}
\hline Group & $n$ & Sex & Age (years) & Weight (kg) \\
\hline REF & 19 & $5 \mathrm{M} / 14 \mathrm{~F}$ & $65.00 \pm 3.62$ & $84.27 \pm 15.93$ \\
EX $_{\mathrm{H}}$ & 16 & $4 \mathrm{M} / 12 \mathrm{~F}$ & $66.3 \pm 6.42$ & $78.79 \pm 19.66$ \\
EX $_{\mathrm{MCI}}$ & 14 & $3 \mathrm{M} / 11 \mathrm{~F}$ & $66.00 \pm 4.79$ & $74.42 \pm 16.45$
\end{tabular}

REF - multimodal training group at the regular exercise facility; $E X_{H}$ - training group of cognitively healthy individuals at the research facility; $E X_{M C I}$ - training group of individuals with mild cognitive impairment at the research facility; Data are mean \pm standard deviation. 
Fig 1. Study Design - Flow Chart: Multimodal trainingprogramme performed at the Regular Exercise Facility

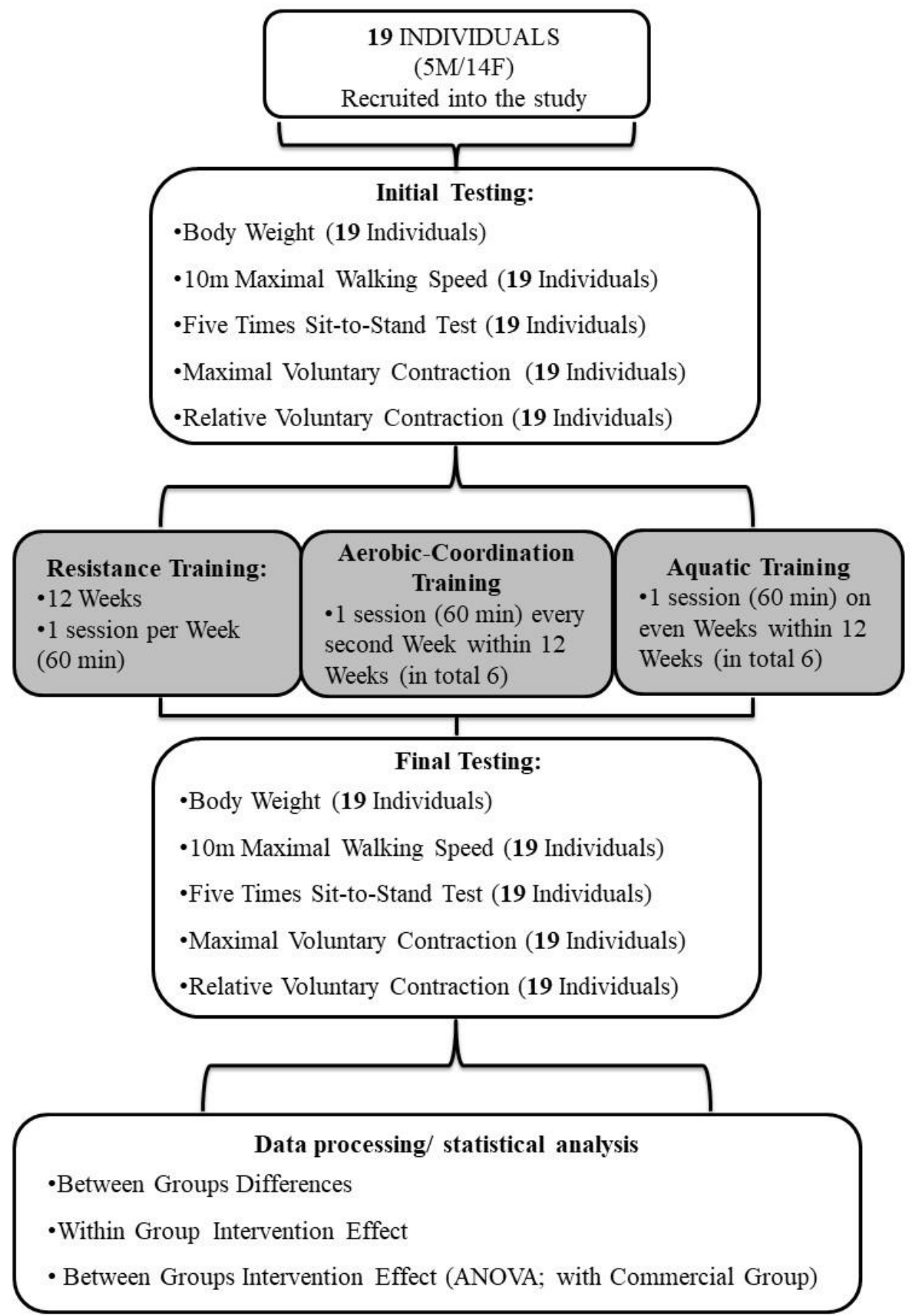

Figure 1a. Study Design - Flow Chart: Multimodal Training Programmme Performed at The Regular Exercise ] Facility 
Fig. 2. Study Design - Flow Chart: Multimodal training programme performed at the Regular Exercise Facility. The dropouts in the post tests were due to non-training relates issues

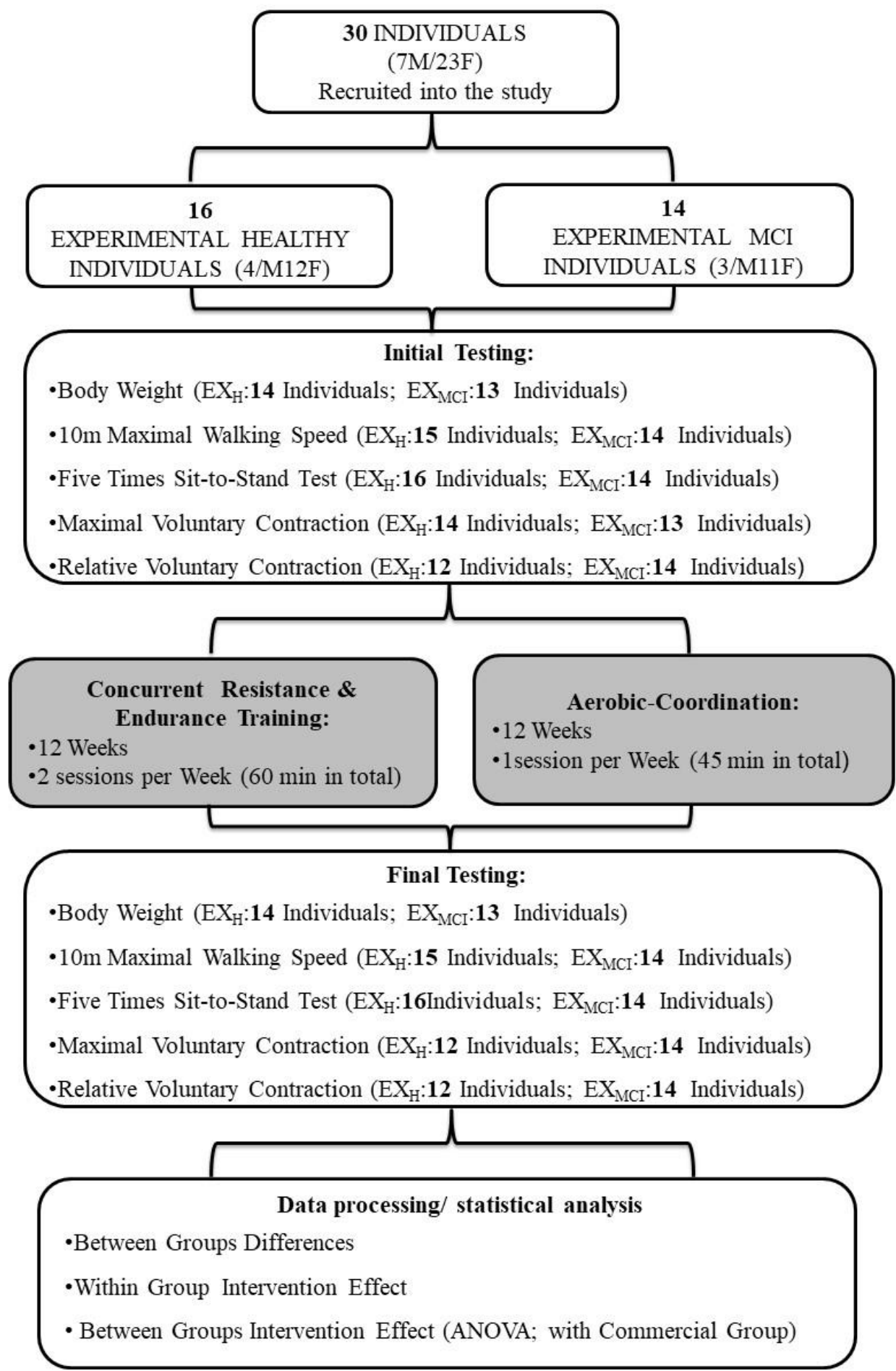

Th Figure 1b. Study Design - Flow Chart: Multimodal Training Programmme Performed at The Research Facility $\mathrm{Th}$

${ }^{\mathrm{co}}$ The dropouts in the post tests were due to non-training related issues. 
individual's ability to perform the current workload. A progression was documented in the exercise diaries. ${ }^{2}$

Aerobic-Coordination Training Session

The aerobic-coordination training sessions lasted 60 minutes each and subjects performed coordination and low-intensity aerobic exercises. The sessions were led in group form. Some exercises included swiss balls, soft gym overballs and steppers as balance equipment. The intensity of exercise represented the aerobic training zone (RPE 13-16 Borg scale). ${ }^{2}$

The Aquatic Training Session

The aquatic training sessions were performed in a pool with water temperature $28^{\circ} \mathrm{C}$, and depth between central chest area and shoulders (impact level 1 and 2). Each training session included shallow water endurance exercises for large muscle groups and aerobic exercises, using aquafitness equipment. The moderate-intensity load with the rating of perceived exertion at the aerobic training zone (RPE 13-16 Borg scale) was used. ${ }^{2}$

12-Week Experimental Multimodal Training Programme, performed at the Research Facility

The multimodal training programme, performed at the research facility, consisted of concurrent resistanceendurance training sessions and aerobic-coordination training sessions. The concurrent resistance-endurance training sessions were performed twice per week in a duration of 60 minutes. The aerobic-coordination training session was performed once per week and lasted 60 minutes too. Within week one and two, subjects performed only one concurrent resistance - endurance and one aerobic-coordination session per week. Following nine weeks, another concurrent resistance endurance training session was added. Thus, participants took part in two concurrent resistance - endurance training sessions and one aerobic-coordination session per week. ${ }^{2,18}$ Professional coaches supervised the training process. All sessions included warm-up (10 minutes), the resistance exercises (approximately 25 minutes), Nordic Walking (18-22 minutes) cool down (approximately 10 minutes), when elderly walked one lap $(380 \mathrm{~m})$ by their preferred walking speed to cool down. ${ }^{10,18}$

Resistance Part

A resistance part of the resistance - endurance training session involved four exercises, focused on large muscle groups, in 2 (first 2 weeks) and 3 sets (following 9 weeks) with the load of 10-12 RM. The tempo of exercises was 2/0/2/1 Inter-set rest interval was 120 seconds. The first session we used for setting individual baseline training loads. Progressive overload of adding loads (2-5\% of estimated 1RM) was used on a weekly basis according to the individual's ability to perform the current workload. A progression was documented in the exercise diaries. $2,10,18$

Endurance Part

Within the endurance part of resistance - endurance training session, elderly performed Nordic Walking (distance: 1750-2100m) with the intensity of 90-95\% of pace measured in pre-intervention Rockport Walk test. Progressive intensity increase according to individual's walking speed, was used in the fifth and ninth week (Week 1.-4.: 1700m, 90\% pace of Rockport test; Week 5.-8.: 2100m 90\% pace of Rockport test; Week 9.-12.: $2100 \mathrm{~m}, 95 \%$ pace of Rockport test). ${ }^{10,18}$

The Aerobic-Coordination Training Session

Each aerobic-coordination session lasted 60 minutes and elderly performed exercises including low aerobics. The sessions were led in group form. Only a stepper was used as balance equipment. The exercise intensity was in a range of aerobic training zone (RPE 13-16 Borg scale). The aerobic-coordination component primarily included changes in direction as well as alternations of left and right side within the performed aerobic composition. ${ }^{2,10,18}$

\section{Measurements}

\section{Meters Test for Maximal Walking Speed}

Volunteers were asked to walk as fast as they can without any assistance. Time to walk over 10 meters was measured electronically by a wireless photocell timekeeping system (FiTRO Light Gates, FiTRONIC, Slovakia) followed by walking speed $(\mathrm{m} / \mathrm{s})$ calculation. MWS was evaluated by performing three trials and the best of performed trials was used for further analyses. The walking technique was controlled visually by researchers and participants were instructed that one foot must always be in touch with the ground. Subjects were not verbally encouraged within the performance of the test. $^{19}$

\section{Times Sit-to-Stand Test}

A standardized testing chair with a straight back and without armrest $(43 \mathrm{~cm}$ seat height, regardless of the height of tested subject), was placed against the wall for safety. Volunteers started from a sitting position, with knees bent at $90^{\circ}$-degree angle, upper backs against the back of the chair and arms crossed over their chest, to a complete stand-up position and return to sitting position. The time (s) of 5 following repetitions within a trial was measured.$^{20}$ The FTSS was performed twice, and the best result was used. Within the performance of the test, the elderly were not verbally encouraged.

The Maximal and Relative Voluntary Contraction in Isometric Knee Extension

MVC in an isometric extension of the knee joint was tested on an adjustable isometric dynamometric chair (S2P Ltd., Ljubljana, Slovenia) adapted for individual anthropometrical parameters of the subjects. Three trials of the bilateral isometric extension were performed. The rest interval was set at 1 minute between each trial. A trial with the highest produced MVC was used. Participants were verbally encouraged within the whole duration of the test. A trial with the highest force produced was taken for further analyses using ARS (Analysis \& Reporting Software, S2P Ltd., Ljubljana, Slovenia). The maximal 
Table 2. Pre to Post induced values within groups and main effect of time

\begin{tabular}{|c|c|c|c|c|c|c|c|c|c|c|}
\hline & \multicolumn{9}{|c|}{ Within-group changes } & \multirow{3}{*}{$\begin{array}{c}\begin{array}{c}\text { Main } \\
\text { Effect }\end{array} \\
\text { Time } \\
\begin{array}{c}(P- \\
\text { value })\end{array} \\
\end{array}$} \\
\hline & \multicolumn{3}{|c|}{ REF } & \multicolumn{3}{|c|}{$\mathbf{E X}_{\mathbf{H}}$} & \multicolumn{3}{|c|}{$\mathbf{E X}_{\text {MCI }}$} & \\
\hline & Pre & Post & $\begin{array}{c}(\mathbf{P}- \\
\text { value })\end{array}$ & Pre & Post & $\begin{array}{c}(\mathbf{P}- \\
\text { value })\end{array}$ & Pre & Post & $\begin{array}{c}(\mathbf{P}- \\
\text { value })\end{array}$ & \\
\hline $\begin{array}{c}10 \mathrm{~m} \\
\mathrm{MWS} \\
(\mathrm{m} / \mathrm{s})\end{array}$ & $\begin{array}{c}2.16 \pm \\
0.26\end{array}$ & $\begin{array}{l}2.36 \pm \\
0.32 *\end{array}$ & .026 & $\begin{array}{c}1.99 \pm \\
0.33\end{array}$ & $\begin{array}{c}2.14 \pm \\
0.37\end{array}$ & .090 & $\begin{array}{c}2.07 \pm \\
0.28\end{array}$ & $2.16 \pm 0.23$ & .061 & .001 \\
\hline FTSS (s) & $\begin{array}{c}9.98 \pm \\
2.12\end{array}$ & $\begin{array}{c}9.13 \pm \\
2.68\end{array}$ & .104 & $\begin{array}{c}10.28 \pm \\
1.31\end{array}$ & $\begin{array}{c}8.85 \pm \\
1.38 * * *\end{array}$ & .000 & $\begin{array}{c}9.91 \pm \\
1.69\end{array}$ & $\begin{array}{c}8.63 \pm \\
1.24 *\end{array}$ & .012 & .000 \\
\hline $\begin{array}{l}\text { MVC } \\
(\mathrm{N} / \mathrm{m})\end{array}$ & $\begin{array}{c}263.66 \\
\pm \\
80.38\end{array}$ & $\begin{array}{c}309.88 \pm \\
83.39 * *\end{array}$ & .002 & $\begin{array}{r}202.62 \\
\pm 42.64\end{array}$ & $\begin{array}{c}226.41 \pm \\
47.39 *\end{array}$ & .018 & $\begin{array}{c}220.40 \pm \\
54.94\end{array}$ & $\begin{array}{c}243.23 \pm \\
45.84 *\end{array}$ & .040 & .000 \\
\hline $\begin{array}{c}\text { rel. MVC } \\
\left(\mathrm{N} / \mathrm{m} \mathrm{kg}^{-2}\right)\end{array}$ & $\begin{array}{c}3.18 \pm \\
0.91\end{array}$ & $\begin{array}{l}3.76 \pm \\
0.86 * *\end{array}$ & .002 & $\begin{array}{c}2.93 \pm \\
0.90 \\
\end{array}$ & $\begin{array}{l}3.42 \pm \\
1.10 * *\end{array}$ & .004 & $\begin{array}{c}3.13 \pm \\
0.90\end{array}$ & $3.54 \pm 0.83$ & .055 & .000 \\
\hline
\end{tabular}

REF - multimodal training group at the regular exercise facility; EXH - training group of cognitively healthy individuals at the research facility; EXMCI -training group of individuals with mild cognitive impairment at the research facility; $10 \mathrm{~m}$ MWS - 10 meters maximal walking speed; FTSS ( $\mathrm{s})$ - five time sit-to-stand test; MVC (N/m) maximal voluntary contraction; rel. MVC relative voluntary contraction;

Data are presented as mean \pm standard deviation; ** indicates significance at $p<0.01$ within group.

MVC of the participant was divided by his or her actual weight to get the relative MVC.

\section{Statistical Analyses}

All statistical analyses were realised in SPSS for Windows (SPSS version 23.0; IBM Corp., Armonk, NY). Conventional statistical methods were used to obtain means, standard deviation (SD). The KolmogorovSmirnov test and Shapiro-Wilk test were used to test normality and Levene's test was used to analyse homogeneity of variance. Possible baseline betweengroup differences were assessed for three groups using a one-way analysis of variance (ANOVA). ANOVA with repeated measures (RMANOVA) was applied to analyse the intervention effects using a 3 group $\left(\mathrm{REF}, \mathrm{EX}_{\mathrm{H}}\right.$, $\left.\mathrm{EX}_{\mathrm{MCI}},\right) \times 2$ time $(\mathrm{PRE}, \mathrm{POST})$ design. Any significant main effect was assessed by Bonferroni post hoc tests for within-group differences. Significance was defined as $p<.05 .^{22}$

\section{Results}

\section{Meters Maximal Walking Speed}

There was a significant main effect of time for 10mMWS $(F=11.970, p=.001)$. Within-group changes, statistically significant increase of walking speed was observed only in the REF group (from $2.16 \pm 0.26 \mathrm{~m} / \mathrm{s}$ to $2.36 \pm 0.32$ $\mathrm{m} / \mathrm{s}, t=-2.431, p=.026$ ) (Table 2.). According to delta changes, no statistically significant changes were observed in any group (Table 3).

\section{Times Sit to Stand Test}

The significant main effect of time was observed in FTSS $(\mathrm{F}=19.866, p=.000)$. Within-group changes, statistically significant decrease of time for FTSS test was observed in $\mathrm{EX}_{\mathrm{H}}$ group (from $10.28 \pm 1.31 \mathrm{~s}$ to $8.85 \pm 1.38 \mathrm{~s}$, $\mathrm{t}=5.201, p=.001$ ) and $\mathrm{EX}_{\mathrm{MCI}}$ group (from $9.40 \pm 1.37 \mathrm{~s}$ to $8.63 \pm 1.24 \mathrm{~s}, t=2.936$; $p=.012$ ) (Table 2.). According to delta changes, statistically significant changes were observed in no group (Table 3.).

\section{Maximal and Relative Voluntary Contraction}

Maximal and relative MVC demonstrated significant main effects for time (MVC: $F=20.148, p=.001$; rel. MVC: $F=22.734, p=.001)$. Within group changes, the significant increase of MVC was observed in the REF group (from 263.66 $\pm 80.38 \quad \mathrm{~N} / \mathrm{m}$ to $309.88 \pm 83.39 \mathrm{~N} / \mathrm{m}, t=3.528, p=.002$ ), $\mathrm{EX}_{\mathrm{H}}$ group (from $202.52 \pm 42.84 \mathrm{~N} / \mathrm{m}$ to $226.41 \pm 47.39 \mathrm{~N} / \mathrm{m}, t=-2.771$, $p=.018$ ) and $\mathrm{EX}_{\mathrm{MCI}}$ group (from $220.40 \pm 54.9484 \mathrm{~N} / \mathrm{m}$ to $243.3 \pm 45.84 \mathrm{~N} / \mathrm{m}, t=2.276, p=.040$ ). Further, the relative MVC increased in the REF group (from $3.18 \pm 0.91 \mathrm{~N} / \mathrm{m} . \mathrm{kg}-2$ to $3.76 \pm 0.86 \mathrm{~N} / \mathrm{m} . \mathrm{kg}-2, t=-3.553$, $p=.002$ ) and the $\mathrm{EX}_{\mathrm{H}}$ group (from $2.93 \pm 0.90 \mathrm{~N} / \mathrm{m} . \mathrm{kg}-2$ to $3.42 \pm 1.10 \mathrm{~N} / \mathrm{m} . \mathrm{kg}-2, t=-3.793, \quad p=.004) \quad($ Table 2). According to delta changes, statistically significant changes were not observed in any training group (Table 3 ). The improvements in the 10mMWS, FTSS, MVC and relative $\mathrm{MVC}$, as indicators of physical performance, indicate better low limbs strength, more stable balance, mobility and neuromuscular function in cognitively healthy elderly. Moreover, the improvement in the FTSS test and absolute MVC in patients with MCI, indicating stronger lower limbs, better coordination and neuromuscular function, might affect the cognitive performance and brain health. ${ }^{19,20,23}$ 


\begin{tabular}{|c|c|c|c|c|}
\hline \multicolumn{5}{|c|}{ Training Groups } \\
\hline & REF & $\mathbf{E X}_{\mathbf{H}}$ & EXMCI & (P-value) \\
\hline$\triangle 10 \mathrm{~m}$ MWS $(\mathrm{m} / \mathrm{s})$ & $0.20 \pm 0.36$ & $0.15 \pm 0.31$ & $0.13 \pm 0.26$ & .820 \\
\hline$\triangle$ FTSS (s) & $-0.85 \pm 2.17$ & $-1.43 \pm 1.10$ & $-0.77 \pm 0.98$ & .451 \\
\hline$\triangle \mathrm{MVC}(\mathrm{N} / \mathrm{m})$ & $46.22 \pm 57.11$ & $34.29 \pm 60.26$ & $22.84 \pm 37.54$ & .463 \\
\hline$\triangle$ rel. MVC $\left(\mathrm{N} / \mathrm{m} \cdot \mathrm{kg}^{-2}\right)$ & $0.57 \pm 0.70$ & $1.13 \pm 1.33$ & $0.56 \pm 0.87$ & .221 \\
\hline \multicolumn{5}{|c|}{$\begin{array}{l}R \overline{E F}-\text { multimodal training group at the regular exercise facility; EXH - training group of cognitively healthy } \\
\text { individuals at the research facility; EXMCI - training group of individuals with mild cognitive impairment at the } \\
\text { research facility; } \triangle 10 \mathrm{~m} M W S \text { - pre to post induced change in maximal walking speed for } 10 \text { meters; } \triangle \text { FTSS - pre to } \\
\text { post induced change in five times sit-to-stand-test; } \triangle M V C \text { - pre to post induced change in maximal voluntary } \\
\text { contraction, } \triangle \text { rel. MVC - pre to post induced change in relative voluntary contraction; }\end{array}$} \\
\hline Data are presented as mec & deviation. & & & \\
\hline
\end{tabular}

\section{Discussion}

In this work, we primarily aimed to compare the effectiveness of the multimodal training that was applied in real life with the Intention-To-Treat strategy and the multimodal training applied at a research centre to previously untrained cognitively healthy elderly. A secondary aim was to identify any specific differences in the training outcomes on walking speed, muscle strength and agility (defining some of the aspects of physical performance) in cognitively healthy elderly and MCI patients According to the results, both types of multimodal training programmes sufficiently improved indicators of physical performance in the elderly. Moreover, the experimental training programme was effective to generate benefits for both cognitively healthy elderly and patients with MCI.

\section{Specificity of multimodal exercise interventions}

Based on strong scientific evidence, it may be assumed that regular multimodal exercise combining many components from resistance, endurance, coordination, flexibility and functional training for around 60 minutes a day, 2 to 3 days per week is sufficient to improve various aspects of physical functioning such as lower limb strength, mobility, balance or walking endurance. ${ }^{1,2,7,8}$ According to overall evidence, the duration of multimodal training programmes varies from 8 weeks to 15 months. However, the most significant effect on the physical performance may be obtained between the ninth and sixteenth week. ${ }^{7}$ According to the duration of training sessions, no apparent differences have been identified in these parameters. ${ }^{2,7}$

For general strength, some trials suggest that 30 minutes of specific resistance training in 12RM could lead to significant strength improvement. ${ }^{24}$ However, among the other trials that incorporated multimodal training, those that reported no significant increase tended to use a shorter total training duration per session (non- significant: $\quad 30-60$ minutes; significant: $75-120$ minutes). ${ }^{7,25,26}$

Both our training protocols partly follow the outcomes, as mentioned earlier. The length of each multimodal training programme was twelve weeks, thus long enough to obtain the noticeable efficacy of the physical performance aspects in previously untrained healthy and MCI elderly. Furthermore, the overall duration of each training session was 60 minutes, and they were performed on two and three non-consecutive days per week. In REF group, the resistance training session lasted only 60 minutes in total and was performed once a week, in 2 to 3 series with the load $10-12 \mathrm{RM}$. The endurance training session was also conducted once a week in 60 minutes alternating the aerobic-coordination and aquatic training every week. However, the time of the session was sufficient to improve aspects of physical performance such and strength of lower limbs Similar results have also been obtained in both cognitively healthy and MCI groups where the multimodal, concurrent resistance - endurance training session consisted of 30 minutes of four resistance exercises in 23 series with the load of 10-12 RM and 20 minutes of Nordic Walking as an endurance component. This type of training session was performed twice a week. In addition, there was an additional aerobic-coordination training session lasting 45 minutes. ${ }^{2,10,18}$ Here, also significant improvements in the five-times-sit-to-stand test, maximal and voluntary contraction in cognitive healthy elderly and patients with mild cognitive impairment occurred.

Effects of multimodal training on physical performance indicators in healthy and MCI elderly

Several studies have shown beneficial effects of physical exercise on mobility and physical performance in MCI elderly.,27,28 Moreover, exercises benefits executive, cognitive and memory in elderly with cognitive decline. ${ }^{7}$ However, most of the studies focused on the 
improvement of physical performance and mobility compared only to individuals with Alzheimer disease or other types of dementia. ${ }^{27,28}$ Baker et al. have reviewed the potential benefits of multimodal training by including 15 randomized controlled trials, totalling 2149 subjects (mean cohort age range from $67 \pm 8$ years to $84 \pm 3$ years). ${ }^{29}$ The authors concluded that multimodal training had a positive effect on the prevention of falls and improving balance. However, limited data available suggested a small effect on physical, functional outcomes and quality of life. ${ }^{7,27,28,29,30}$ Also our findings show that the frequency and structure of both presented multimodal training could improve in different range the indicators of physical performance. Also, changes in the physical performance predict brain plasticity changes, but they may not always translate to cognitive functions. However, to elicit the detectable cognitive effects, the should last longer than three months (>6 months).7 Although, the further research must be done to explore the sufficient duration, dosage and intensity of exercise that are needed to improve the cognitive function in MCI elderly. According to our study, de Oliviera Silva et al. has shown similar outcomes to our study. 12-week multimodal training programme performed twice a week in MCI elderly significantly improved mobility and executive functions. ${ }^{30}$ The elderly MCI improved in the simple task 8-feet up and go test compared to controls $(\mathrm{t}=2.28 ; p=.03)$. Our findings confirm these outcomes and show that 12-week experimental multimodal training programme performed three times per week is sufficient to improve parameter of physical performance such as maximal walking speed, and maximal relative voluntary contraction in patients with MCI. The $\mathrm{EX}_{\mathrm{MCI}}$ training group significantly improved in five-times-sit-to-stand test and maximal voluntary contraction. There was also observed improvement in relative voluntary contraction. Moreover, there were no significant differences between healthy and MCI elderly after finishing the training programme. Thus we may conclude that the 12 -week experimental multimodal training is effective in previously untrained MCI elderly, as well as in cognitively healthy elderly. The strength of the study is identifying and comparing the effectiveness of the intention-to-treat or real-life intervention strategy performed twice per week (one resistance session and one aerobic-coordination session and alternating aquatic session) with the experimental protocol, performed three times per week (two resistance-endurance sessions and one aerobic-coordination session) as a part of the research program, while the various aspects defining physical performance were determined. Moreover, an identification of the experimental multimodal training's effectiveness on various aspects of physical performance in patients with MCI is of additional value. There are also some limitations to the study. The general limitations are the variability in design, possible differences in subjects' motivation (paid vs free-of-charge programme) small samples of participants, especially in the experimental groups as well as the slight statistical heterogeneity of the groups. Moreover, providing MCI patients with the reallife Intention-To-Treat intervention would also be very interesting in the future. From a practical point of view, our findings could be useful for coaches and medical practitioners who work with the elderly. The results demonstrated that there is no difference in the efficacy between multimodal training performed twice a week with the real-life Intention-To-Treat strategy and similar experimental training with the frequency three times per week provided as a part of the experimental research project. Both training strategies were sufficient to improve indicators of physical performance in cognitively healthy elderly, thus may indirectly improve overall health and quality of life for the elderly. $7,19,20,23$ If we compare the efficiency, the REF multimodal training performed twice a week might be a more suitable choice for both the business and clinical sphere. Moreover, experimental multimodal training can increase physical performance in patients with MCI. We might hypothesize that this type of multimodal training benefits not only for physical performance but the neurocognitive performance and brain health of patients with MCI due to the improvement of mobility and muscle strength, which are associated with changes in brain structures. ${ }^{13}$ In conclusion, both modes of multimodal training might be a key factor to make the elderly more active and support their adherence to the active lifestyle and their healthy ageing. However, further research should be done to explore how multimodal training affects physical performance and brain health in patients with MCI.

\section{List of acronyms}

10mMWS - 10 meters maximal walking speed

$\mathrm{EX}$ - experimental training programmme

$\mathrm{EX}_{\mathrm{H}}$ - experimental healthy group

$\mathrm{EX}_{\mathrm{MCI}}$ - experimental mild cognitive impaired group

FTSS - five times sit-to-stand test

MCI - mild cognitive impairment

MMSE - mini-mental state examination

MoCA - Montreal cognitive assessment

MVC - maximal voluntary contraction

$\mathrm{REF}$ - regular exercise facility

$\mathrm{RM}$ - repetition maximum

RPE - rating of perceived exertion

$\mathrm{SD}$ - standard deviation

\section{Authors contributions}

LO, LS, MS wrote the manuscript, LO, MV, AK, VT, JC, $\mathrm{GB}, \mathrm{MS}$ designed and performed the real-life multimodal exercise-intervention program in the elderly. BU \& JU designed the study evaluating effects of exercise in cognitively healthy and MCI patients. LO, LS, MV, VT, MS contributed to the training process and data collection. LO, LS, MS analysed and interpreted data. All authors contributed to the critical reading and final editing of the manuscript. 


\section{Acknowledgments}

We want to thank all volunteers who took part in the study, the entire research team and the coaches who helped us.

\section{Funding}

The study was supported by Slovak Research \& Development Agency SRDA15-0253 (BU), Grant Agency of the Slovak Academy of Sciences VEGA 2/0107/18 (BU), Comenius University students grants UK/336/2018, UK/402/2019 and via the international cooperation scheme INTEREG V-A Slovakia - Austria Centre of Active Ageing - competency-based centre for physical activity, prevention and health promotion of seniors (acronym CAA, ITMS2014+ 305041X157) funded by European Regional Development Fund

\section{Conflict of Interest}

The authors have no conflicts to disclose.

\section{Ethical Publication Statement}

We confirm that we have read the Journal's position on issues involved in ethical publication and affirm that this report is consistent with those guidelines.

\section{Corresponding Author}

A/prof. Milan Sedliak, PhD. Faculty of Physical Education and Sport, Department of Biological and Medical Sciences, Comenius University in Bratislava Nábr. arm. gen. L. Svobodu 9, 81469 Bratislava, Slovakia. ORCID iD: 0000-0002-2324-154X

E-mail: milan.sedliak@uniba.sk

\section{E-mails of co-authors}

Ludmila Oreská: ludmila.oreska@uniba.sk ORCID iD: 0000-0002-2963-7924

Lucia Slobodová: lslobodova77@gmail.com ORCID iD: 0000-0001-5465-6257

Matej Vajda: matej.vajda@uniba.sk ORCID iD: 0000-0002-1471-8465

Adriana Kaplánová: adriana.kaplanova@gmail.com ORCID iD: : 0000-0001-7548-5805

Veronika Tirpáková: veronika.tirpakova@uniba.sk ORCID iD: 0000-0003-1142-0214

JánCvečka:myofitsk@gmail.com ORCID iD: 0000-0001-6555-642X

Gabriel Buzgó: gabriel.buzgo@uniba.sk ORCID iD: 0000-0001-7712-7358

Jozef Ukropec: jozef.ukropec@savba.sk ORCID iD: 0000-0001-8401-6621

Barbara Ukropcová: barbara.ukropcova@savba.sk ORCID iD: 0000-0002-3309-7713

\section{References}

1. Sbardelotto ML, Costa RR, Malysz KA, et al. Improvement in muscular strength and aerobic capacities in elderly people occurs independently of physical training type or exercise model. Clinics
(Sao Paulo). 2019;74:1-9. doi:10.6061/clinics/ 2019/e833.

2. Sousa N, Mendes R, Abrantes C, et al. Effectiveness of combined exercise training to improve functional fitness in older adults: A randomized controlled trial. Geriatr Gerontol Int 2014;14:892-8. doi: 10.1111/ggi.12188.

3. Izquierdo M, Häkkinen K, Ibañez J, et al. Effects of strength training on muscle power and serum hormones in middle-aged and older men. J. Appl. Physiol. 2001;90:1497-1507. doi: 10.1152/ jappl.2001.90.4.1497.

4. Landi F, Onder G, Carpenter I, et al. Physical activity prevented functional decline among frail community-living elderly subjects in an international observational study. J Clin Epidemiol 2007;60:518-24. doi: 10.1016/j.jclinepi.2006.09. 010.

5. Lopez P, Pinto RS, Radaelli R, et al. Benefits of resistance training in physically frail elderly: A systematic review. Aging Clin Exp Res 2018;30: 889-899. doi: 10.1007/s40520-017-0863-z.

6. Peterson MD, Rhea MR, Sen A, et al. Resistance exercise for muscular strength in older adults: a meta-analysis. Ageing Res Rev 2010;9:226-37. doi: 10.1016/j.arr.2010.03.004.

7. Lam FMH, Huang MZ, Liao LR, et al. Physical exercise improves strength, balance, mobility, and endurance in people with cognitive impairment and dementia: A systematic review. J Physiother 2018;64:4-15. doi: 10.1016/j.jphys.2017.12.001.

8. Pitkälä K, Savikko N, Poysti M, et al. Efficacy of physical exercise intervention on mobility and physical functioning in older people with dementia: a systematic review. Exp Gerontol 2013;48:85-93. doi: $10.1016 / \mathrm{j}$.

9. Furtado HL, Sousa N, Simão R, et al. Physical exercise and functional fitness in independently living vs institutionalized elderly women: a comparison of 60- to 79-year-old city dwellers. Clin Interv Aging 2015;10:795-801. doi:10.2147/CIA. S80895

10. Máderová D, Krumpolec P, Slobodová L, et al. Acute and regular exercise distinctly modulate serum, plasma and skeletal muscle BDNF in the elderly. Neuropeptides 2019;78.101961.

11. Sanders LMJ, Hortobágyi T, la Bastide-van Gemert $\mathrm{S}$, et al. Dose-response relationship between exercise and cognitive function in older adults with and without cognitive impairment: A systematic review and meta-analysis. PLoS One 2019;14:e 0210036. doi:10.1371/journal.pone.0210036.

12. Haskell WL, Lee IM, Pate RR, et al. Physical activity and public health: updated recommendation for adults from the American College of Sports Medicine and the American Heart Association. Med Sci Sports Exerc 2007;39:1423-34. doi: 10.1249 /mss.0b013e3180616b27

13. Nelson ME, Rejeski WJ, Blair SN, et al. Physical 
activity and public health in older adults: recommendation from the American College of Sports Medicine and the American Heart Association. Med Sci Sports Exerc 2007;39:143545. doi: 10.1249/mss.0b013e3180616aa2.

14. Farlie M. K, Robins L, Haas R, et al. Programme frequency, type, time and duration do not explain the effects of balance exercise in older adults: a systematic review with a meta-regression analysis. Br J Sports Med 2018;53:996-1002. 10.1136/ bjsports-2016-096874

15. Phillips EM, Kennedy MA. The exercise prescription: A tool to improve physical activity. PM R 2012;4:818-25.

16. Bouaziz W, Lang PO, Schmitt E, et al. Health benefits of multicomponent training programmes in seniors: a systematic review. International Journal of Clinical Practice 2016;70:520-36. doi: 10.1111/ ijcp. 12822.

17. Schon M, Slobodova L, Tirpakova V, et al. A Link Between Cognitive Function and Physical Activity: the Impact of Aerobic-Strength Exercise in Seniors With Mild Cognitive Impairment and/or Impaired Glucose Metabolism. Alzheimer's \& Dementia, 2018;14. P1030.

18. Krumpolec P, Vallova S, Slobodova L, et al. AerobicStrength Exercise Improves Metabolism and Clinical State in Parkinson's Disease Patients. Front Neurol 2017;8:698. doi:10.3389/fneur.2017.00698.

19. Kim DH, Kim CA, Placide S, et al. Preoperative Frailty Assessment and Outcomes at 6 Months or Later in Older Adults Undergoing Cardiac Surgical Procedures: A Systematic Review. Ann Intern Med 2016;165:650-60. doi:10.7326/M16-0652

20. Makizako H, Shimada H, Doi T, et al. Predictive Cutoff Values of the Five-Times Sit-to-Stand Test and the Timed "Up \& Go" Test for Disability Incidence in Older People Dwelling in the Community. Physical therapy 2017;97:417-24. doi: 10.2522/ptj.20150665

21. Sarabon N, Rosker J, Fruhmann H, et al. Reliability of maximal voluntary contraction related parameters measured by a novel portable isometric knee dynamometer. Phys Med Rehab Kuror 2013;23: 22 - 7. doi: 10.1055/s-0032-1331190

22. Ihalainen JK, Inglis A, Mäkinen T, et al. Strength Training Improves Metabolic Health Markers in Older Individual Regardless of Training Frequency. Front Physiol 2019;10:32. doi:10.3389/fphys.

\subsection{2}

23.Guadagnin EC, Priario LAA, Carpes FP, Vaz MA. Correlation between lower limb isometric strength and muscle structure with normal and challenged gait performance in older adults. Gait and Posture 2019;73:101-7. doi: 10.1016/j.gaitpost.2019.07. 131.

24. Bossers WJ, van der Woude LH, Boersma F, et al. A 9-week aerobic and strength training program improves cognitive and motor function in patients with dementia: a randomized, controlled trial. Am J Geriatr Psychiatry 2015;23:1106-16. doi: 10.1016 /j.jagp.2014.12.191.

25. Arcoverde C, Deslandes A, Moraes H, et al. Treadmill training as an augmentation treatment for Alzheimer's disease: a pilot randomized controlled study. Arq Neuropsiquiatr 2014;72:190-6. doi: 10.1590/0004-282X20130231.

26. Netz Y, Axelrad S, Argov E. Group physical activity for demented older adults feasibility and effectiveness. Clin Rehabil 2007;21:977-86. doi: 10.1177/0269215507078318.

27. Liang JH, Xu Y, Lin L, et al. Comparison of multiple interventions for older adults with Alzheimer disease or mild cognitive impairment: A PRISMA-compliant network meta-analysis. Medicine (Baltimore). 2018;97:e10744. doi:10. 1097/MD.0000000000010744.

28. Northey JM, Cherbuin N, Pumpa KL, et al. Exercise interventions for cognitive function in adults older than 50: a systematic review with meta-analysis. $\mathrm{Br}$ J Sports Med 2018;52:154-60. doi: 10.1136/ bjsports-2016-096587.

29. Baker M, Kennedy D, Bohle P, et al. Efficacy and feasibility of a novel tri-modal robust exercise prescription in a retirement community: a randomized, controlled trial. J Am Geriatr Soc 2007;55:1-10.

30. de Oliveira Silva F, Ferreira JV, Plácido J, et al. Three months of multimodal training contributes to mobility and executive function in elderly individuals with mild cognitive impairment, but not in those with Alzheimer's disease: A randomized controlled trial. Maturitas 2019;126:28-33. doi: 10.1016/j.maturitas.2019.04.217.

Submissions: January 13, 2020

Revision received: Febraury 20, 2020

Acceptance: Febraury 21, 2020 